\title{
Rheumatic fever at the end of the twentieth century: Why is there still a problem?
}

\author{
Edward L. Kaplan
}

$\Lambda$ $t$ the beginning of the final decade of the 20th century, the issues relating to health care for the world's children are incredibly complex. The mixture of the "same old problems" and the devastating new ones, in the face of a chronic lack of resources made even worse by both natural and man-made catastrophes, seems to have cast an even darker shadow across the faces of children and their advocates. Unfortunately, having neither the vote nor the funds necessary to influence the political process, it appears that children and children's causes suffer even more. Faced with an epidemic of incredible proportions and universal lethality on the one hand, and these "usual childhood" infectious diseases and malnutrition on the other, governments and international public health agencies have great difficulties in taking purposeful action that can have a documented impact. The truth is that, although the names of the diseases and perhaps their relative magnitude may change from country to country, the issues related to children's welfare and health remain present, no matter whether one speaks of industrializing or industrialized countries. Health care for the world's children has become increasingly frustrating!

While examples of these diseases are most often referred to in terms of infectious diseases and nutrition, morbidity and mortality due to cardiovascular diseases in the pediatric population are no different. In fact, these cardiovascular diseases may even be more frustrating because there is so much that remains unknown about their immediate and long-term effects. For example, how does one address the consequences of children's diet on subsequent development of cardiovascular disease in many parts of the world where malnutrition and starvation are a fact of everyday life? What will be the magnitude decades into the future of the effects of smoking in countries that apparently have been targeted by multinational companies for promotion of tobacco?

With enormous problems and limited resources, cost effectiveness becomes more and more important. Al- though nobody wants to minimize new approaches to medical or surgical treatment of disease, the vocabulary of preventive approaches is more frequently heard in both official and in non-governmental planning councils. An excellent example of this has been the World Health Organization's Expanded Program on Immunization. This program has made vaccines against common childhood infections such as measles and polio available to countries where local governmental resources simply cannot do so. Such a preventive approach is simply not only practical, but it has proven realistic!

If these facts are true (and the confirming evidence is overwhelming), then, when physicians and other health care professionals concerned with the cardiovascular health of children ask why the greater part of an issue of Cardiology in the Youngshould be devoted to rheumatic fever and rheumatic heart disease, the argument can be made that this is a preventable disease, and that programs for prevention have already been demonstrated to be cost-effective by the World Health Organization and others. These cardiovascular sequels to infection of the upper respiratory tract by the group A beta hemolytic streptococcus, however, remain very significant problems around the world today. Astounding figures for prevalence are available from many countries. Recent data published by the World Health Organization note prevalence rates of rheumaticheart disease of up to 20/ 1,000 schoolchildren in some countries. Even worse, in many countries in which there is reason to believe that there is a significant problem, very few data are even available.

The counter argument is frequently made that it is clear that this disease is declining in frequency, and sooner or later it will almost disappear as it has largely in the western industrialized countries of North America and Europe, and in some countries of Asia such as Japan. The recent and incompletely explained "resurgence" of rheumatic fever in the United States of America, nonetheless, provides evidence that antibiotics and increased standard of living and increased avail- 
ability of health care cannot totally control the problem. Of more immediate concern, published data from some countries reveal that, in the latter decades of the twentieth century, the largest cardiovascular problem in the pediatric population is rheumatic in origin. It may be three or even four times more common in children presenting for medical care than is congenital heart disease. For many of these countries it is obvious that adequate resources and facilities for surgery of rheumatic valvar disease are not available. Furthermore, antibiotics such as penicillin for primary and secondary prevention of rheumatic fever are also often in short supply. Of interest is the fact that, even when antibiotics are available, they may not be used appropriately or their manufacture may not always be quality controlled. It has been said that rheumatic fever and its subsequent heart disease is the most controllable of major cardiovascular diseases in the pediatric (and perhaps the adult) population, and yet it remains a significant problem in countries which, in fact, make up a majority of the world's population.

Decades after the realization that prompt antibiotic therapy of the preceding streptococcal infection can essentially eliminate the threat of rheumatic fever, major questions remain unanswered about this problem. What is it about the group A streptococcus that makes it "rheumatogenic"? There are intriguing hypotheses currently being explored in the basic science laboratory, but the truth of the matter is that, in 1992, we do not know! For decades it has been recognized by astute clinicians and epidemiologists that there seems to be a genetic or familial predisposition to developing rheumatic fever, but again the truth of the matter is that, in 1992, we do not know! Secondary prevention of recurrent attacks of rheumatic fever by continuous administration of penicillin is very effective as a public health program. Primary prevention is also effective in preventing initial attacks in individual patients, but the practical applicability of this latter approach in children in developing countries, with often times marginal health care, has not been sufficiently studied to result in implementation of public health programs. Even where there are supportive data for such programs, because of competing priorities in countries with limited resources, the programs have not even been tested.

The recent application of molecular biology to the understanding of the structure and function of the group A streptococcus has led to promising beginnings for the development of an effective vaccine, but many questions remain to be answered about the efficacy of such a vaccine, even in the experimental animal models.
Clinical vaccine trials will have to be undertaken with caution since questions about the offending responsible antigenic moiety of the organism also remain unanswered.

And all the while, despite important advances resulting from clinical, epidemiological, basic and applied research, this cardiovascular disease continues to be a significant public health problem in countries around the world. In this issue of Cardiology in the Young, some of the current issues relating to rheumatic fever and rheumatic heart disease are thoughtfully discussed by recognized authorities from several countries of the world where rheumatic fever and rheumatic heart disease remain major problems. The recognition of the need to understand more completely the pathogenesis of the cardiac involvement of rheumatic fever is thoughtfully addressed. The long-term natural history of rheumatic heart disease, and the difficulties in its diagnosis, remain important problems for the clinician. Similarly, controversy still exists about both the advantages and the limitations of both medical and surgical therapy of rheumatic fever and rheumatic heart disease. One of the recently introduced diagnostic techniques has been the echocardiogram. Long-term and prospective observations to understand the findings in both rheumatic fever and rheumatic heart disease are necessary for the clinician, and have been presented in two papers in this issue.

These discussions serve to bring this very significant problem into focus again, and to remind pediatricians and pediatric cardiologists of an "old problem" that remains unsolved at the end of the 20th century. Furthermore, it serves as a pointed reminder that considerably more research is needed in this area in order to adequately address the problem. One important conclusion reached after studying this issue of Cardiology in the Young is that this research can be successfully carried out in those countries where the disease is most prevalent. Furthermore, it can be argued that clinicians and scientists there have both the opportunity and the obligation to further the understanding of this enigmatic disease, and then to implement these advances into practical techniques for improving the cardiovascular health of a significant proportion of children in the world today.

\author{
Box 296 UMHC \\ Department of Pediatrics \\ University of Minnesota Medical School \\ 420 Delaware St SE \\ Minneapolis, Minnesota 55455, USA \\ Fax. 612-624-8927
}

